\title{
Distribución de la riqueza de especies de los odonatos (Insecta: Odonata) de la República Dominicana: una actualización a partir de especímenes de colección
}

\author{
Distribution of species richness of the odonates (Insecta: Odonata) of the Dominican Republic: An \\ update from collection specimens
}

\author{
América Sánchez-Rosario
}

\begin{abstract}
Resumen
Las colecciones científicas, como la del Instituto de Investigaciones Botánicas y Zoológicas (IIBZ) de la Universidad Autónoma de Santo Domingo, son importantes registros de la biodiversidad. Éstas ofrecen una visión parcial, aunque útil, de las localidades típicas de incidencia de los seres vivos y de los vacíos de información. En este artículo se presenta una lista de especies y un mapa de incidencia de los odonatos en estado adulto representados en la colección del IIBZ, República Dominicana, y se realiza una comparación con reportes de trabajos previos. De las 67 especies registradas actualmente en República Dominicana, 63 fueron encontradas en la colección. Respecto a trabajos previos, la incidencia de especies aumentó en varias provincias, destacando el caso del Distrito Nacional, que aumentó de 44 a 51 especies. Un análisis de correlación evaluó la asociación de la riqueza numérica con la densidad de drenaje, la precipitación anual y la superficie de la provincia. Ninguna de las correlaciones resultó significativa. Adicionalmente se generaron modelos lineales de efecto mixto, utilizando como efecto fijo las variables mencionadas y, como efecto aleatorio, una variable cualitativa relacionada con la accesibilidad y la densidad poblacional. Los modelos encontraron que un porcentaje considerable de la varianza de la riqueza numérica es explicado por el efecto aleatorio, lo que significa que la accesibilidad condiciona la incidencia de especies, sugiriendo que existe sesgo de muestreo. Se plantea la necesidad de realizar recolectas en regiones con pocos registros para conocer mejor la distribución de los odonatos en el país.
\end{abstract}

Palabras claves: estadística zonal, precipitación, provincia, red hidrográfica, sesgo de muestreo, libélulas

\begin{abstract}
Scientific collections are important biodiversity records, as is the one from the Instituto de Investigaciones Botánicas y Zoológicas (IIBZ) from the Universidad Autónoma de Santo Domingo in Dominican Republic. They provide a partial but useful view of the common occurrence localities and information gaps of specific groups of organisms. Presented in this paper are a list of species and an occurrence map of adult odonate specimens represented in the collection from the IIBZ; a comparison of them with reports from previous works was performed. Of the 67 species currently reported in the Dominican Republic, 63 were found in the collection. Regarding previous works, species richness increased in some provinces, such was the case in Distrito Nacional, where it increased from 44 to 51 . A correlation analysis examined the association between numeric richness and the drainage density, annual precipitation and surface area of the province; none of the correlations yielded significant results. Additionally, linear mixed effect models were generated, using the mentioned
\end{abstract}

Instituto de Investigaciones Botánicas y Zoológicas Prof. Rafael M. Moscoso, Universidad Autónoma de Santo Domingo, Santo Domingo, República Dominicana.

* Autor de correspondencia: <america.sanchez@hotmail.com> 
variables as fixed effect and a qualitative variable related to accessibility and population density as random effect. All the models found that a substantial percentage of the variance of the numeric richness is explained by the random effect variable, which means the accessibility conditions the species' occurrence, suggesting a sampling bias exists. From this arises the need of collecting specimens in regions with few records to better comprehend the distribution of odonates in the country.

Keywords: zonal statistics, precipitation, province, drainage network, sampling bias, dragonflies

\section{INTRODUCCIÓN}

Los registros de especímenes de colecciones científicas son de gran importancia, pues representan un respaldo científico y cultural muy valioso por el material biológico que resguardan (Simmons y Muñoz-Saba 2005). Con el estudio de los taxones, en las colecciones, se obtiene información acerca de la fauna registrada en distintas regiones y se estima la distribución de las especies en un determinado hábitat (Sánchez-Cordero et al. 2001).

Diversos estudios han puesto de manifiesto que, en décadas recientes, la biodiversidad en la República Dominicana ha estado sometida a múltiples amenazas que determinan la destrucción de hábitats, como el fuego, la tala, la modificación de áreas para la agricultura y la ganadería, entre otras (Fern y Moreno 2015, Kennedy et al. 2006, Myers et al. 2004), lo cual ha aumentado el riesgo de pérdida de diversidad.

En comparación con otras islas del Caribe, el territorio dominicano presenta una diversidad singular de hábitats y de especies, tanto por su compleja historia geológica (Pérez-Estaún et al. 2010) como por su paleogeografía (Graham 2003, Pindell y Kennan 2009), factores que afectan la riqueza numérica y la distribución de especies. Por otra parte, la hidrografía del país también es muy variable; concretamente, el caudal de los cursos fluviales varía considerablemente según la elevación, extensión y pluviosidad de sus respectivas cuencas en las provincias (De la Fuente 1976). Por lo anterior, la República Dominicana se considera como un lugar idóneo en el Caribe para estudios de diversidad de distintos grupos en general y, en particular, de insectos como los odonatos.

Aunque en República Dominicana se han realizado estudios sobre taxonomía y distribución de odonatos (Bastardo y Sánchez-Rosario 2017, Flint et al. 2006), es importante integrar la información, actualizando y completando las listas de especies según su distribución geográfica en el país, objetivo principal de este estudio. Dado que la evaluación sobre el estado del conocimiento y la distribución de la riqueza de especies puede indicar cuáles son las áreas prioritarias para estudios de conservación (De Marco y Vianna 2005), el presente artículo constituye un punto de partida para priorizar áreas de muestreo. En tal sentido, se presenta una lista y mapa de distribución de los odonatos en estado adulto, representados en la colección del Instituto de Investigaciones Botánicas y Zoológicas (IIBZ) de la Universidad Autónoma de Santo Domingo (UASD), República Dominicana.

\section{MATERIALES Y MÉTODOS}

Se analizaron registros de incidencia por provincias a partir de 2.196 especímenes adultos con datos completos de la colección de odonatos del IIBZ, los cuales representan aproximadamente el $80 \%$ del total de ejemplares de la colección y abarcan el periodo de recolección entre 1969-2018. Con el objetivo de evaluar la evolución de la lista de especies por provincias, dichos registros se compararon con los publicados por Flint et al. (2006).

Los especímenes de la colección fueron registrados en una hoja de cálculo de LibreOffice Calc 5.4, donde se transcribieron y depuraron los datos contenidos en las etiquetas, con especial atención en la localidad, coordenadas geográficas y determinación taxonómica. Los especímenes sin determinación a nivel de especie fueron identificados utilizando las claves de Needham et al. (2000) y Westfall y May (1996).

Los registros de incidencia aportados por Flint et al. (2006) se basaron en la división provincial vigente a la fecha de su estudio (ONE 1998). Sin embargo, desde entonces, la división provincial experimentó 
cambios (ONE 2017) que obligan a tomar determinadas decisiones para hacer posible la comparación de los valores de riqueza. Dado que la provincia San José de Ocoa fue escindida de Peravia, los registros recientes de la primera fueron agregados a los de la segunda. Una estrategia similar se adoptó con respecto a la provincia Santo Domingo, la cual surgió luego de segregar el anterior Distrito Nacional. Finalmente, como la provincia Salcedo cambió de nombre, sus registros fueron atribuidos a su más reciente denominación, Hermanas Mirabal.

Adicionalmente, se evaluó si existe relación de la riqueza numérica de especies por provincias con tres variables ambientales seleccionadas. Se evaluó el grado de asociación de la riqueza con la densidad de drenaje de la red hidrográfica de orden cuatro o superior, con la media de la precipitación provincial y la superficie de la provincia. Para evaluar el grado de asociación de la riqueza con las variables seleccionadas, se utilizó la técnica estándar del coeficiente de correlación de Pearson y, adicionalmente, se generaron modelos lineales de efecto mixto. Para construir los modelos de efecto mixto se utilizaron las variables mencionadas como efecto fijo y, como efecto aleatorio, se utilizó una variable categórica para clasificar las provincias en distintos grupos en función de su densidad de carreteras/calles y su densidad poblacional. Se evaluaron distintos modelos, considerando varias opciones de agrupamiento de las provincias, incluyendo o excluyendo el valor atípico correspondiente al Distrito Nacional (DN). Como modelo idóneo se eligió aquel con el menor valor de AIC.

Para calcular la densidad de drenaje, primero se construyó la red hidrográfica nacional utilizando el modelo digital de elevaciones (MDE) de la misión SRTM, con resolución 3 arco-segundos (NASA LP DAAC 2000). Posteriormente, la longitud de cursos por provincia fue sumada y dividida entre la superficie provincial, obteniéndose así la densidad de drenaje.

Como fuente de datos de precipitación se utilizó el compendio WorldClim Version 2 (Fick y Hijmans 2017), el cual consiste en promedios mensuales para el periodo 1970-2000, dada en milímetros por metro cuadrado, servidos en superficies continuas de 1 kilómetro cuadrado de resolución. Mediante álgebra de mapas, primero se sumaron las superficies mensuales $\mathrm{y}$, posteriormente, por medio de estadística zonal, se obtuvieron las medias provinciales.

Las tareas de limpieza, transformación y análisis de los datos se realizaron en el ambiente de programación R ( $\mathrm{R}$ Core Team 2015). El mapa y la estadística zonal se realizaron utilizando el software QGIS (QGIS Development Team 2018). La recogida de datos se llevó a cabo en el laboratorio del IIBZ.

\section{RESULTADOS}

De las 67 especies reportadas para la isla por Flint et al. (2006), 63 están presentes en la colección del IIBZ (tabla 1). De estas 63, las de mayor incidencia fueron: Orthemis ferruginea (Fabricius, 1775) presente en 24 provincias, Enallagma coecum (Hagen, 1861) reportada en 21 provincias, y Erythrodiplax umbrata (Linnaeus, 1758) y Macrothemis celeno (Selys, 1857) presentes en 20 y 18 provincias respectivamente.

La figura muestra que varias provincias centro occidentales, centro orientales y septentrionales, presentan baja riqueza numérica de especies respecto del promedio provincial, tanto en el estudio de 2006 como en la revisión de la colección del IIBZ. Por otra parte, el número de especies desde 2006 hasta 2018 aumentó considerablemente en varias provincias tras la revisión realizada en el presente estudio (figura y tabla 2). En primer lugar, algunas provincias de mayor riqueza aumentaron considerablemente su número de especies. En el estudio de Flint et al. (2006), Distrito Nacional tenía 44 especies, mientras que en la colección actualmente hay registradas 51 (máxima riqueza provincial y máximo incremento absoluto), lo que equivale a un incremento de más de un $15 \%$ respecto del valor anterior. Asimismo, Barahona aumentó de 27 a 32 especies, Monseñor Nouel de 24 a 29, Puerto Plata de 21 a 25 y Dajabón de 9 a 14. En segundo lugar, varias provincias aumentaron su riqueza con al menos 3 especies en promedio, como Distrito Nacional, Independencia y María Trinidad Sánchez. En tercer lugar, provincias que anteriormente no contaban con registros, como 
Para las tablas y la figura se usará la siguiente clave de provincias (según ONE, 1998): AL = La Altagracias, AZ $=$ Azua, $\mathrm{BH}=$ Bahoruco, $\mathrm{BR}=$ Barahona, $\mathrm{CR}=$ San Cristóbal, DA = Dajabón, DN = Distrito Nacional, $\mathrm{DU}=$ Duarte, EP = Elías Piña, ES = Espaillat, HI = Hermanas Mirabal, HM = Hato Mayor, IN = Independencia, JU $=$ San Juan, $\mathrm{MC}=$ Monte Cristi, $\mathrm{MN}=$ Monseñor Nouel, MP = Monte Plata, MT = María Trinidad Sánchez, $\mathrm{PM}=$ San Pedro de Macorís, $\mathrm{PN}=$ Pedernales, $\mathrm{PP}=$ Puerto Plata, $\mathrm{PR}=$ Peravia, $\mathrm{RO}=\mathrm{La}$ Romana, $\mathrm{SE}=\mathrm{El}$ Seibo, SM = Samaná, SR = Santiago Rodríguez, ST = Santiago, SZ = Sánchez Ramírez, VA = Valverde, VE $=$ La Vega.

Tabla 1. Distribución de especies por provincia, actualización a partir de la colección del IIBZ combinado con los reportes de Flint et al. (2006). Aquellas especies que aún no han sido registradas en esta colección se encuentran marcadas con un asterisco $\left({ }^{*}\right)$

\begin{tabular}{|c|c|c|c|c|c|c|c|c|c|c|c|c|c|c|c|c|c|c|c|c|c|c|c|c|c|c|c|c|c|c|}
\hline Especies & $\mathrm{AL}$ & $\mathbf{A Z}$ & $\mathrm{BH}$ & BR & CR & DA & DN & DU & EP & ES & $\mathrm{HM}$ & IN & JU & MC & $M N$ & MP & MT & PM & PN & PP & PR & RO & $\mathrm{HI}$ & SE & SM & SR & ST & SZ & VA & VE \\
\hline Anax amazili & - & - & - & - & - & - & - & - & - & - & - & - & - & + & - & - & - & - & - & - & - & - & - & - & - & - & - & - & - & + \\
\hline $\begin{array}{l}\text { Anax } \\
\text { concolor* }\end{array}$ & - & - & - & - & - & - & + & - & - & - & - & - & - & - & + & - & - & - & - & - & - & - & - & - & - & - & - & - & - & + \\
\hline Anax junius & - & - & - & - & - & - & + & - & - & - & - & - & - & - & + & - & - & - & - & - & - & - & - & - & - & - & - & - & - & - \\
\hline $\begin{array}{l}\text { Aphylla } \\
\text { caraiba }\end{array}$ & - & - & - & - & + & - & + & - & - & - & - & + & - & - & + & - & - & - & - & + & + & - & - & - & + & - & - & + & + & + \\
\hline $\begin{array}{l}\text { Brachymesia } \\
\text { furcata }\end{array}$ & - & - & - & + & - & - & + & - & - & - & - & - & - & - & + & - & - & - & - & - & + & - & - & - & - & - & - & - & - & - \\
\hline $\begin{array}{l}\text { Brachymesia } \\
\text { herbida }\end{array}$ & - & - & - & + & - & - & + & - & - & - & - & - & - & - & + & - & - & - & - & - & - & - & - & - & + & - & - & - & - & + \\
\hline $\begin{array}{l}\text { Cannaphila } \\
\text { insularis }\end{array}$ & - & - & - & + & + & - & - & - & - & - & - & + & - & - & + & - & - & - & - & + & - & - & - & - & + & - & + & - & - & + \\
\hline $\begin{array}{l}\text { Coryphaeschna } \\
\text { adnexa }\end{array}$ & - & - & - & + & - & - & + & - & - & - & - & - & - & - & - & - & - & - & - & - & - & - & - & - & - & - & + & + & - & + \\
\hline $\begin{array}{l}\text { Coryphaeschna } \\
\text { viriditas }\end{array}$ & - & + & - & - & - & - & + & - & - & - & - & - & - & - & - & - & - & - & - & + & - & - & - & - & + & - & + & - & - & + \\
\hline $\begin{array}{l}\text { Diceratobasis } \\
\text { melanogaster }\end{array}$ & - & - & - & - & - & - & + & - & - & - & - & - & - & - & + & - & + & - & - & - & - & - & - & - & - & - & - & - & - & + \\
\hline $\begin{array}{l}\text { Dythemis } \\
\text { rufinervis }\end{array}$ & - & - & - & + & + & + & + & + & + & - & - & - & + & - & - & - & + & - & + & - & + & - & + & + & + & - & + & + & + & + \\
\hline $\begin{array}{l}\text { Enallagma } \\
\text { civile }\end{array}$ & - & + & - & + & - & + & + & - & - & - & - & - & - & + & - & - & - & - & + & + & - & - & - & + & + & - & - & - & - & + \\
\hline $\begin{array}{l}\text { Enallagma } \\
\text { coecum }\end{array}$ & - & + & + & + & + & + & + & + & + & + & + & + & + & + & + & + & + & - & + & + & + & - & - & + & + & - & + & + & + & + \\
\hline $\begin{array}{l}\text { Erythemis } \\
\text { attala* }\end{array}$ & - & - & - & - & - & - & - & - & - & - & - & - & - & - & - & - & - & - & - & - & - & - & - & - & - & - & - & - & - & - \\
\hline $\begin{array}{l}\text { Erythemis } \\
\text { plebeja }\end{array}$ & - & + & - & + & + & - & + & - & + & - & - & - & - & - & - & - & + & - & - & - & + & + & - & + & - & - & - & - & - & + \\
\hline $\begin{array}{l}\text { Erythemis } \\
\text { simplicicollis }\end{array}$ & - & - & + & + & - & - & - & - & - & - & - & - & - & - & - & - & - & - & - & - & - & - & - & - & + & - & - & - & - & - \\
\hline $\begin{array}{l}\text { Erythemis } \\
\text { vesciculosa }\end{array}$ & + & + & - & + & - & - & + & - & - & - & - & + & - & + & + & - & + & - & + & + & - & + & - & + & - & - & + & - & + & + \\
\hline $\begin{array}{l}\text { Erythrodiplax } \\
\text { berenice }\end{array}$ & - & - & - & + & - & - & + & - & - & - & - & - & - & - & - & - & - & - & + & + & + & - & - & - & - & - & - & - & - & - \\
\hline $\begin{array}{l}\text { Erythrodiplax } \\
\text { fervida }\end{array}$ & - & - & - & + & + & - & + & - & - & - & - & - & - & - & + & - & - & - & - & + & - & - & - & - & + & - & - & - & - & + \\
\hline $\begin{array}{l}\text { Erythrodiplax } \\
\text { justiniana }\end{array}$ & - & + & - & + & + & - & + & - & - & - & + & + & - & - & + & - & + & - & - & - & + & - & - & - & + & - & + & - & - & + \\
\hline $\begin{array}{l}\text { Erythrodiplax } \\
\text { umbrata }\end{array}$ & + & + & - & + & - & + & + & + & + & - & - & + & + & + & + & + & + & + & + & + & + & + & - & + & + & - & + & + & + & + \\
\hline $\begin{array}{l}\text { Gynacantha } \\
\text { nervosa }\end{array}$ & + & - & + & - & + & - & + & - & - & - & - & - & - & - & - & + & + & - & - & - & - & + & - & + & + & - & + & + & - & + \\
\hline $\begin{array}{l}\text { Hypolestes } \\
\text { hatuey }\end{array}$ & - & + & - & + & + & - & + & + & - & + & + & - & - & - & + & + & + & - & + & + & - & - & - & - & + & - & - & + & - & + \\
\hline $\begin{array}{l}\text { Idiataphe } \\
\text { cubensis }\end{array}$ & - & + & - & - & - & - & - & - & - & - & - & - & - & - & - & - & - & - & - & - & + & - & - & - & - & - & - & - & - & - \\
\hline
\end{tabular}




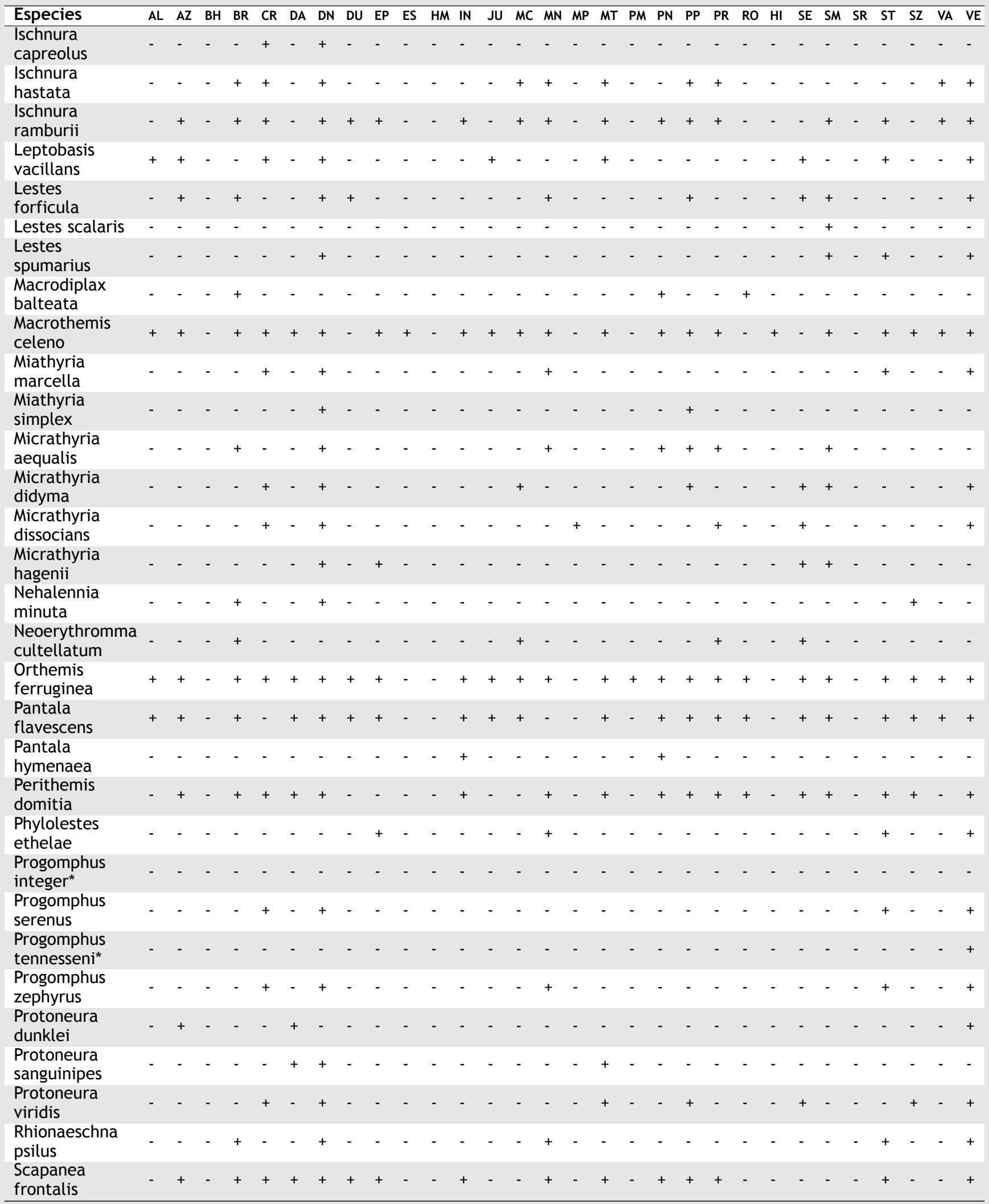




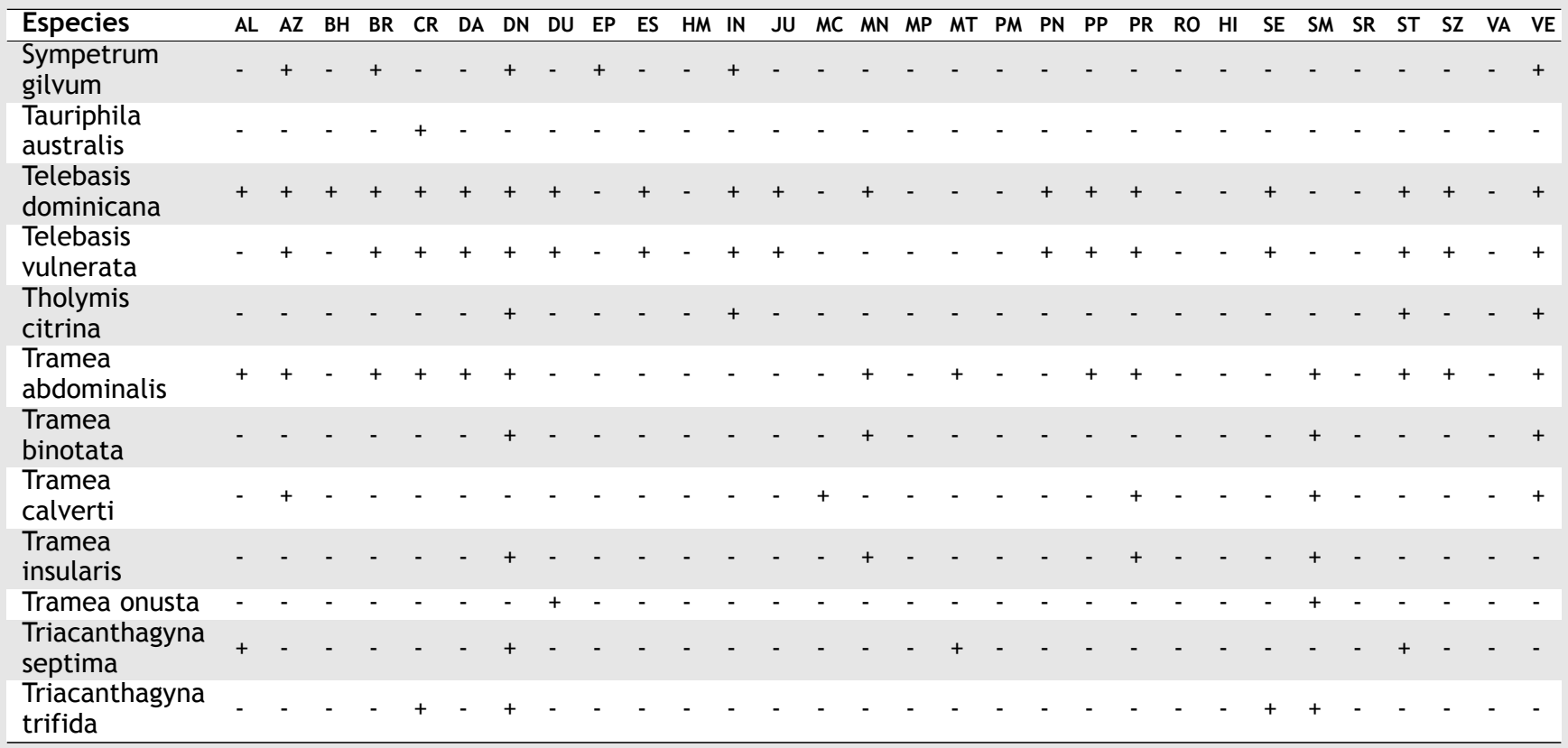

No reportada: - Reportada: +

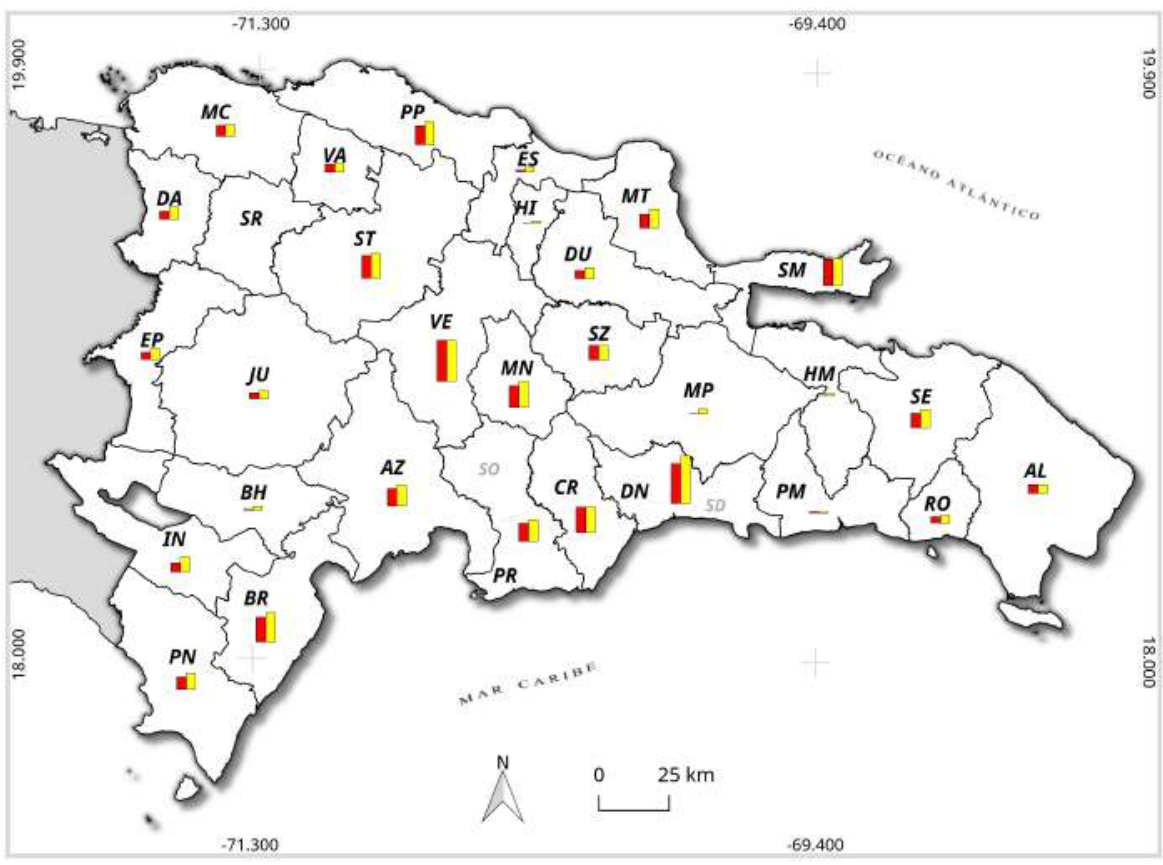

Figura. Representación geográfica de la riqueza reportada para 2006 y los registros encontrados en la colección del IIBZ por provincia en la República Dominicana.

Polígonos de borde negro = provincia; línea gris = límites de la provincia San José de Ocoa y Distrito Nacional según división vigente (ver detalles en el texto); barras de color rojo = número de especies según registros hasta 2006; barras de color amarillo = número de especies acumulado (incluye registros del IIBZ). 
Tabla 2. Riqueza de especies, precipitación promedio, densidad de drenaje y área por provincia.

\begin{tabular}{|c|c|c|c|c|c|}
\hline $\begin{array}{l}\text { Provin- } \\
\text { cia }\end{array}$ & $\begin{array}{l}\text { Flint } \\
\text { et al. } \\
\text { (2006) }\end{array}$ & $\begin{array}{l}\text { Riqueza } \\
2018\end{array}$ & PP (mm) & D & $\mathrm{A}\left(\mathrm{km}^{2}\right)$ \\
\hline $\mathrm{AL}$ & 10 & 10 & 1100,474 & 0,077 & 2995,911 \\
\hline$A Z$ & 19 & 23 & 766,257 & 0,096 & 2682,540 \\
\hline $\mathrm{BH}$ & 1 & 4 & 709,942 & 0,125 & 1285,007 \\
\hline $\mathrm{BR}$ & 27 & 32 & 803,898 & 0,070 & 1659,945 \\
\hline CR & 28 & 28 & 1293,203 & 0,107 & 1240,811 \\
\hline DA & 9 & 14 & 1075,651 & 0,083 & 1021,251 \\
\hline DN & 44 & 51 & 1438,122 & 0,099 & 1393,158 \\
\hline DU & 9 & 12 & 1531,049 & 0,119 & 1649,479 \\
\hline EP & 8 & 12 & 1238,942 & 0,146 & 1395,473 \\
\hline ES & 2 & 5 & 1343,704 & 0,061 & 842,440 \\
\hline $\mathrm{HM}$ & 0 & 3 & 1483,523 & 0,099 & 1319,146 \\
\hline IN & 10 & 17 & 848,747 & 0,101 & 1770,951 \\
\hline JU & 7 & 9 & 1024,379 & 0,105 & 3363,808 \\
\hline$M C$ & 12 & 13 & 745,479 & 0,132 & 1885,814 \\
\hline$M N$ & 24 & 29 & 1374,022 & 0,103 & 992,033 \\
\hline MP & 0 & 5 & 1694,670 & 0,102 & 2601,616 \\
\hline MT & 15 & 21 & 1569,624 & 0,094 & 1206,503 \\
\hline PM & 2 & 2 & 1078,465 & 0,086 & 1253,998 \\
\hline PN & 14 & 18 & 837,651 & 0,058 & 2080,413 \\
\hline PP & 21 & 25 & 1222,232 & 0,075 & 1805,634 \\
\hline PR & 20 & 24 & 972,486 & 0,085 & 1637,985 \\
\hline RO & 7 & 8 & 923,425 & 0,066 & 651,754 \\
\hline $\mathrm{HI}$ & 0 & 2 & 1302,569 & 0,002 & 427,386 \\
\hline SE & 16 & 20 & 1298,308 & 0,077 & 1788,324 \\
\hline SM & 29 & 30 & 1752,744 & 0,060 & 862,732 \\
\hline SR & 0 & 0 & 1035,926 & 0,106 & 1147,545 \\
\hline ST & 25 & 27 & 1079,416 & 0,126 & 2806,560 \\
\hline SZ & 16 & 16 & 1644,284 & 0,137 & 1185,803 \\
\hline VA & 9 & 10 & 886,319 & 0,137 & 822,983 \\
\hline VE & 45 & 45 & 1125,473 & 0,101 & 2292,651 \\
\hline
\end{tabular}

PP: precipitación promedio A: área D: densidad

Hermanas Mirabal, Hato Mayor y Monte Plata tienen al menos dos especies registradas en la colección del IIBZ. Contrario a lo anterior, destaca el caso de la provincia Santiago Rodríguez, la única sin registros de odonatos tanto en el estudio de 2006 como en la colección del IIBZ.

Por último, utilizando los registros de incidencia combinados de las listas de 2006 y de 2018 , se determinó que no existe correlación de la riqueza numérica de especies con la precipitación promedio $(r=0,11$, $p=0,58)$ o con la densidad de drenaje $(r=0,03, p$ $=0,86)$, ni con la superficie provincial $(r=0,15, p=$ $0,43)$.

Los modelos lineales de efecto mixto mostraron, en todos los casos, varianzas explicadas por el efecto aleatorio entre el $10 \%$ (modelos elaborados excluyendo el DN) y $65 \%$ (modelos elaborados incluyendo el DN). El modelo que obtuvo el menor AIC (228) presentó una varianza explicada por efecto aleatorio asociado a densidad de calles y densidad poblacional de un $30 \%$. Este modelo utilizó, como variables independientes de efecto fijo, la precipitación, la densidad de drenaje y la superficie provincial, siendo esta última la única que obtuvo un coeficiente positivo y significativo, para un nivel de significancia de 0,05.

\section{DISCUSIÓN}

La baja riqueza numérica de especies de algunas provincias centro occidentales, centro orientales y septentrionales se interpreta como un sesgo en el esfuerzo de muestreo a través del tiempo, un hecho que afecta a colecciones de éste y otros grupos en distintos países (p. ej., De Marco y Vianna 2005, Sánchez-Cordero et al. 2001).

Algunos estudios indican que los especímenes en las colecciones biológicas son, en gran medida, tomados de manera oportunista, lo cual se traduce en más muestras tomadas cerca de vías de acceso (Gernandt et al. 2014, Pyke y Ehrlich 2010, Sánchez y Martínez 2017). La ausencia de registros en la provincia Santiago Rodríguez, cuya accesibilidad es reducida, refuerza esta idea del sesgo en favor de sitios accesibles y próximos a los centros donde se localizan las entidades de investigación. Adicionalmente, esta singular ausencia de registros en Santiago Rodríguez, justifica la necesidad de realizar exploraciones en dicha provincia, así como en otras que tradicionalmente han sido poco estudiadas, como algunas centrooccidentales, centro-orientales y septentrionales del país (p. ej., Bahoruco, Hato Mayor, Monte Plata, San Pedro de Macorís y Hermanas Mirabal).

Por otra parte, según el análisis de correlación y el modelo lineal de efecto mixto seleccionado, la densidad de drenaje y la precipitación no parecen asociarse con la riqueza de especies. Asimismo, el modelo atribuye cierta relación positiva de la riqueza con la superficie provincial, un resultado inesperado que amerita estudios posteriores.

Por lo tanto, los análisis realizados, sugieren que la riqueza obtenida a partir de los datos de la colección, está influenciada por el sesgo de muestreo, este últi- 
mo atribuible a la accesibilidad por carretera y a la densidad poblacional. Por tal razón, la distribución de la riqueza de odonatos por provincias requiere de mayores esfuerzos para obtener mejores estimados de la riqueza por provincia.

Algunos casos destacados por su baja riqueza encontrada, y en los que se esperaría encontrar más especies por su gran superficie y porque han sido poco exploradas, son las provincias Elías Piña, Monte Plata, Hato Mayor, Duarte y, especialmente Santiago Rodríguez, la cual no cuenta con registros en la colección (tabla 2).

Finalmente, como conclusión, el presente estudio revela la importancia de completar las listas de especies según las distintas regiones del país, como primer paso para reducir el sesgo de muestreo existente, lo cual en definitiva contribuiría a seleccionar áreas prioritarias en estudios futuros. Con suerte y esfuerzo, se obtendrá una visión más completa sobre el estado del conocimiento y la distribución de la riqueza de odonatos en la República Dominicana.

\section{AGRADECIMIENTOS}

A los asistentes voluntarios Edwin Félix, Lisa Guerrero, María E. Hernández, Alinhjel García y Laura Montero por ayudar en el proceso de digitalización de las etiquetas. A José Ramón Martínez por proporcionar los scripts para la limpieza de data en $\mathrm{R}$ y corrección del manuscrito. A Ruth Bastardo y Eveling Gabot por sus sugerencias.

\section{CONFLICTO DE INTERESES}

Los autores declaran no tener conflictos de intereses.

\section{REFERENCIAS}

Bastardo RH y Sánchez-Rosario A. 2017. Estado del conocimiento de los macroinvertebrados acuáticos de la isla La Española. Actualidades Biológicas, 39:1-22. doi:10.17533/udea.acbi.v39n107a09

De la Fuente S. 1976. Geografía dominicana. Santo Domingo: Amigo del Hogar. p. 272.

De Marco P y Vianna DM. 2005. Distribuição do esforço de coleta de Odonata no Brasil: subsídios para escolha de áreas prioritárias para levantamentos faunísticos. Lundiana 6 (1): 13-26.

Fern LB y Moreno AH. 2015. Reservas de Biosfera fronterizas en la República de Haití y la República Dominicana (CAReBios). 517 p.

Fick SE y Hijmans RJ. 2017. Worldclim 2: New 1-km spatial resolution climate surfaces for global land areas. International Journal of Climatology. 37 (12): 4302-4315.

Flint OS Jr, Bastardo RH, Perez-Gelabert DE. 2006. Distribution of the Odonata of the Dominican Republic. Bulletin of American Odonatology, 9 (3, 4): 67-84.

Gernandt DS, Chavez GAS, Davila VM, y Héau OJG. 2014 Digitalización del Herbario Nacional de México: avances y retos del futuro. Revista digital universitaria 15(4): 113. http://www.revista.unam.mx/vol.15/num4/art30/

Graham A. 2003. Geohistory models and Cenozoic paleoenvironments of the Caribbean region. Systematic Botany, 28: $378-386$.

Kennedy LM, Horn SP y Orvis KH. 2006. A 4000-year record of fire and forest history from Valle de Bao, Cordillera Central, Dominican Republic. Palaeogeography, Palaeoclimatology, Palaeoecology, 231:279-290. doi:10.1016/j.palaeo.2005.08.00

Myers R, OBrien J. Mehlman D. y Bergh C. 2004. Evaluación del Manejo del Fuego en los Ecosistemas de Tierras Altas de la República Dominicana, 25 p.

NASA LP DAAC. 2000. SRTM 1 Arc-Second Global. NASA EOSDIS Land Processes DAAC, USGS Earth Resources Observation and Science (EROS) Center, Sioux Falls, South Dakota. [fecha de acceso mayo 2016] https: //earthexplorer.usgs

Needham JG, Westfall Jr y May ML. 2000. Dragonflies of north america. p. 940.

Oficina Nacional de Estadística (ONE), 1988. División Territorial de la República Dominicana, 225 p.

Oficina Nacional de Estadística (ONE), 2017. División Territorial de la República Dominicana, 561 p. www.one.gob.do/informaciones-cartograficas/ division-politica-administrativa/ mapa-politico-administrativo

Pérez-Estaún A., Hernaiz Huerta PP, Lopera E, Joubert M, Escuder Viruete J, et al. 2010. Geología de la República Dominicana: de la construcción de arco-isla a la colisión arco-continente. Boletín Geológico y Minero, 118: 157174.

Pindell JL y Kennan L. 2009. Tectonic evolution of the Gulf of Mexico, Caribbean and northern South America in the mantle reference frame: an update. Geological Society, London, Special Publications, 328: 1-55.

Pyke GH y Ehrlich PR. 2010. Biological collections and ecological/environmental research: a review, some observations and a look to the future. Biological reviews, 85(2), 247266.

QGIS Development Team, 2018. QGIS. A Free and Open Source Geographic Information System. Online. [fecha de acceso mayo 2018] https://qgis.org/

R Core Team, 2015. R: A language and environment for statistical computing.

Sánchez-Cordero V, Townsend A, y Escalante P. 2001. El modelado de la distribución de especies y la conservación de 
la diversidad biológica. Enfoques contemporáneos para el estudio de la biodiversidad, 359-379.

Sánchez RA y Martínez B JR. 2017. Cobertura arbórea de los parques urbanos Mirador del Norte y Jardín Botánico Nacional. IX Congreso Internacional Interdisciplinario de Investigación Científica. Libro de Resúmenes, 167 p. https://www.geografiafisica.org/2019/04/27/postercobertura-arborea-de-los-parques-urbanos-mirador-delnorte-y-jardin-botanico-nacional/

Simmons JE y Muñoz-Saba Y. 2005. Cuidado, manejo y conservación de las colecciones biológicas. Univesidad Nacional de Colombia. p. 288.

Westfall MJ y May ML. 1996. Damselflies of North America. Vol. 649. Scientific Publishers Gainesville. p. 650. 